\title{
Frequency/amplitude characteristics of postural tremor of the hands in a population of patients with bilateral essential tremor: implications for the classification and mechanism of essential tremor
}

\author{
STEFANO CALZETTI,* MARIO BARATTI, ${ }^{*}$ MICHAEL GRESTY,$\dagger$ \\ LESLIE FINDLEY $\dagger$
}

From Istituto di Clinica Neurologica, ${ }^{*}$ Universita' di Parma, Parma, Italy, and the MRC Neuro-Otology Unit, National Hospital for Nervous Diseases, $\dagger$ London, UK

SUMMARY Amplitude/frequency characteristics of postural hand tremor in 59 patients with bilateral essential tremor of various degrees of severity were assessed using accelerometric recordings and spectral analysis. Intra-subject comparisons of tremor characteristics between the more and less affected hands were used to control for variability of tremor due to age factors and intersubject differences in amplitude and frequency. Statistical analysis distinguished three different patient groups. Some patients had low amplitude $(<0 \cdot 1-0.015 \mathrm{~cm})$ tremor in the less affected limb (which tended to be $7 \mathrm{~Hz}$ or more in frequency in the young) and a larger amplitude tremor in the more affected hand which was $1 \mathrm{~Hz}$ or more lower in frequency. Other subjects had either bilaterally small or bilaterally large amplitude tremors of similar frequencies. These findings imply that there is a downwards step in frequency between symptomatic tremors of small and large amplitude. The amplitude and frequency of the small amplitude tremors were unrelated but frequency declined with age. The frequency of the large amplitude tremor was generally determined by amplitude but a wide range of amplitudes were compatible with similar frequencies. The frequency of large amplitude tremor also declined with age. It was concluded that there are two types of essential tremor, the smaller amplitude tremor probably derives from an exaggeration of some or all of the mechanisms of normal physiological tremor whereas the larger amplitude tremor probably arises from a separate "pathological" central nervous mechanism. It is not known if or how one may transform into, or be replaced by, the other during progression of the disease.

Tremor of the hands is the most frequent symptom in benign essential tremor (ET), a common disorder of unknown aetiology and sporadic or familal occurrence. ${ }^{1}$ The tremor, at onset, may be unilateral, but almost invariably the tremor becomes bilateral and often other body parts become involved during the progression of the disorder. Although the tremor may be present both on posture and through movement, usually it has been characterised by measurement of the postural component present when the hand is

Address for reprint requests: Dr L Findley, The National Hospital for Nervous Diseases, Queen Square, London WC1N 3BG, UK.

Received 20 June 1986. Accepted 15 August 1986 maintained outstretched in unsupported posture. It is generally accepted that postural ET of the hands shows an inter-subject range distribution of the dominant peak frequency varying between about 4 to $11 \mathrm{~Hz},{ }^{23}$ and that there is an inter-patient trend for tremor frequency to decrease in inverse proportion of the age of the patient and the amplitude of the tremor. ${ }^{4}$ However, these overall population characteristics may mask more complex changes in the behaviour of the tremor during the progression from early to advanced stages.

There are two hypotheses concerning the mechanism of essential tremor. The "unitary mechanism" theory, proposed by Elble ${ }^{5}$ on the basis of group data, is of a central oscillator responsible for syn- 
chronous modulation of motor unit spike trains which is minimally present in normal individuals, but is clearly developed in patients with mild ET, and oscillates at a frequency of 8 to $12 \mathrm{~Hz}$. More severe essential tremor is of a lower frequency $(4$ to $8 \mathrm{~Hz})$ and also dependent upon a central oscillator. As both mild and severe ET can occur in the same family it is inferred that the mechanism is the same for both and that, with progression of the disorder, the frequency of the oscillator decreases. In contrast, Marsden et $a l,{ }^{6}$ on the basis of the behavioural characteristics of individual tremors, attribute ET to at least two different tremor mechanisms. Type I is behaviourally indistinguishable from enhanced physiological tremor, has a frequency of 8 to $12 \mathrm{~Hz}$ and is due to increased gain of the stretch reflex. Type II ET is of lower frequency ( 5 to $7 \mathrm{~Hz}$ ), of larger amplitude, and dependent upon oscillation from within the central nervous system. Unlike type I, type II is resistant to being driven by an external input to the stretch reflex.

So far there have been no longitudinal, prospective studies of the population characteristics of ET which have followed the course of the disease in individual patients to show how the tremor changes with age and severity which might indicate the mechanism(s) involved. An alternative approach, which we have used in this study, is to make intra-subject comparisons of the tremors in the two hands of patients who have bilateral tremor of various degrees of severity. In this way, each subject acts as a control for age and the comparison of the amplitude/frequency relationships in the more and less affected hand should reveal whether or not there is a continuous linear relationship between frequency and amplitude. A continuous proportionality would suggest a unitary tremor mechanism whereas any discontinuity of the linear relationship would favour a plurality of mechanisms.

\section{Methods}

\section{Patients}

Fifty-nine patients with mild to severe ET in both hands (44 male and 15 female), aged between 16 and 82 years (median 59 years) who were attending the out patient clinics at the National Hospital for Nervous Diseases, Queen Square, were studied. All patients had been symptomatic in both hands for at least one year prior to the study (median duration of tremor on the more involved hand: 9 years, range 1-70 years). In 24 patients there was a family history of tremor affecting the hands and/or the head. Fourteen patients who were taking beta-adrenoceptor antagonists or other drugs for tremor at the time of the study, had their treatment discontinued for at least 2 weeks prior to assessment.

\section{Protocol}

The patients were asked to abstain from taking alcohol and caffeinated beverages and from smoking for at least 12 hours before testing and were each examined on one occasion. After 15 minutes rest, allowing the patient to become accustomed to the environment, piezoresistive linear accelerometers (ENDEVCO 7625-10) were taped to the dorsal surface of each hand in the second interspace $1 \mathrm{~cm}$ proximal to the metacarpophalangeal joints, their sensitive axis being orientated in the vertical plane. These devices weighed $6 \mathrm{~g}$ and had a frequency response extending from a steady state acceleration to $300 \mathrm{~Hz}$ with a sensitivity of $50 \mathrm{mV} / \mathrm{g}(\mathrm{g}=$ acceleration of gravity). Hand tremor was assessed with the patient seated, fully relaxed and looking directly ahead. The forearms were supported up to the wrists and the hands were unsupported and outstretched horizontally in a pronated posture during the recording. Three separate recordings of about 1 minute duration were obtained at 5 minute intervals, and to minimise the effect of fatigue the hands were allowed to rest freely between recordings. Accelerometric signals were amplified and recorded simultaneously on paper and magnetic tape for subsequent tremor analysis.

\section{Tremor analysis}

Analysis was performed off-line using a Hewlett-Packard $5420 \mathrm{~A}$ signal analyser. The programme averaged $150 \mathrm{spec}-$ tra with a bandwidth of DC to $25 \mathrm{~Hz}$ and frequency resolution of $0.096 \mathrm{~Hz}$, each derived from overlapping $10.24 \mathrm{sec}-$ ond samples of tremor. Fifty samples were taken from the beginning of each of the three separate recordings and approximately 45 seconds of tremor contributed to the analo ysis of each condition. The average spectrum was displayed for measurement as the root mean squared (rms) magnitude of the frequency components plotted as a function of the frequency. For a simple characterisation of the tremor, meaco surements were taken of the frequency $(\mathrm{Hz})$ of the dominan peak and of its magnitude scaled in rms acceleration, the unit of acceleration being taken as $1 \mathrm{~g}\left(\mathrm{~g}=981 \mathrm{~cm} / \mathrm{s}^{2}\right)$. The conversion from tremor magnitude of acceleration (expressed in milli-g) to actual tremor amplitude (expressed as $\mathrm{cm}$ hand displacement) was calculated by using the formula: amplitude of displacement $=\left(\right.$ acceleration $\times 981 / 4 \pi^{2}$ $x$ frequency $^{2}$ ) $\mathrm{cm}$ rms.

In order to elucidate the mechanisms of tremors of apparently similar frequencies, the coherence function was calculated from the 150 individual spectra as a measure of the degree of causal relationship between the tremor of the two hands at their respective dominant peak frequency. ${ }^{7}$ The coherence takes a value from 0 to 1 , where zero indicates that the two tremor signals are totally unrelated and 1 indicates that the signals are totally causally related, that is, one signal can be considered to be driven entirely from the other or that both derive from a common origin. Figure 1 shows an example of spectra of the tremors of the hands and of their relative coherence in a patient included in the study.

\section{Statistical analysis}

Since tremor amplitude showed a skew distribution, log transformation was made to normalise these data. Pearson's correlation coefficient was used to evaluate the relationship between the continuous variables, that is, dominant peak frequency and amplitude of tremor, age of the patients, age at the onset and duration of the disorder and to compute the relationship between the difference in peak frequency and 

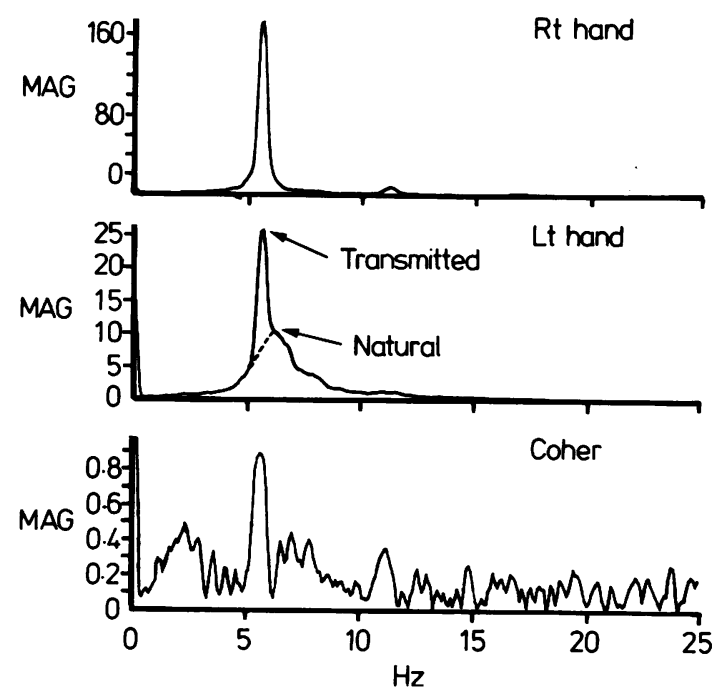

Fig 1 Examples of averaged rms spectra and mutual coherence of postural tremor of the hands of a patient with ET. MAG is tremor magnitude in units of $\mathrm{g} \times 10^{-3}$.

amplitude of tremor of the two hands. For the identification of subgroups of patients according to tremor amplitude, analysis of variance (ANOVA) and Duncan's multiple range test were used.

\section{Results}

Table 1 shows the mean amplitude and peak frequency of tremor in the two hands, the coherence at the dominant peak frequency of each hand (calculated in 46 patients) and the mean difference in tremor amplitude $(\Delta \mathrm{a})$ and in peak frequency $(\Delta \mathrm{f})$ between the two hands in the patients studied.

The distributions of the dominant peak frequency with respect to log amplitude displacement of tremor in the more and in the less affected hands are shown in figs 2 and 3 respectively.

The relationships between tremor characteristics (frequency/amplitude of more/less affected hand) and patient's characteristics (age, age at onset and

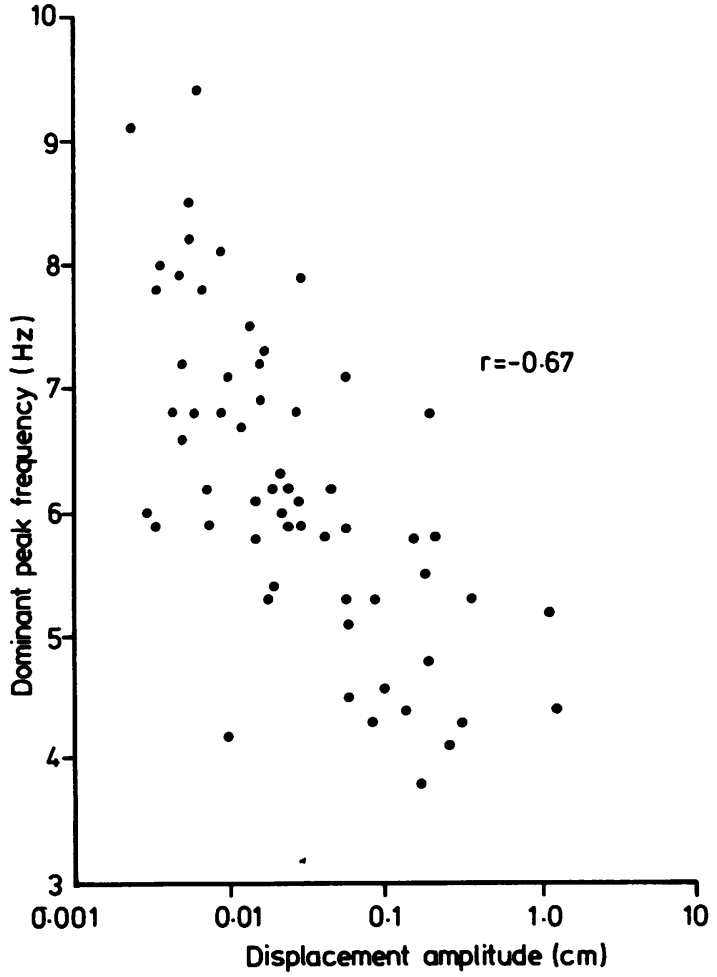

Fig 2 Relationship between dominant peak frequency $(\mathrm{Hz})$ of tremor and log-amplitude displacement (cm) of tremor in the more involved hand in the patients studied $(r=0.67$, $p<0.001$ ).

duration of disorder) are similar to those reported by previous studies (see introduction) and are shown in the correlation matrix of table 2. Relationships of particular note are: the negative correlation between frequency and amplitude expressed by a decrease in tremor peak frequency of approximately $0.6 \mathrm{~Hz}$ on the more affected and 0.7 on the less affected hand respectively for a two fold increase in tremor amplitude and, secondly, the overall significant correlation between the difference in tremor amplitude $(\Delta a)$ between the two hands and their relative difference in

Table 1 Amplitude, frequency and coherence statistics for 59 patients with ET

\begin{tabular}{llll}
\hline & $\begin{array}{l}\text { Tremor amplitude } \\
\text { (cm hand displacement) } \\
\text { mean } \pm S D\end{array}$ & $\begin{array}{l}\text { Tremor dominant } \\
\text { peak frequency }(\mathrm{Hz}) \\
\text { mean } \pm S D\end{array}$ & $\begin{array}{l}\text { Coherence }(0-1) \\
\text { mean } \pm S D\end{array}$ \\
\hline $\begin{array}{l}\text { More involved hand } \\
\text { Less involved hand }\end{array}$ & $\begin{array}{l}0.094 \pm 0.21(\mathrm{~N}=59) \\
0.020 \pm 0.03\end{array}$ & $\begin{array}{l}6.24 \pm 1.29(\mathrm{~N}=59) \\
6.62 \pm 1.32\end{array}$ & $\begin{array}{l}0.35 \pm 0.2(\mathrm{~N}=46) \\
0.24 \pm 0.1\end{array}$
\end{tabular}

Mean difference in tremor amplitude $(\Delta \mathrm{a}): 0.074 \mathrm{~cm}$ (more-less involved hand)

Mean difference in tremor peak frequency $(\Delta): 0.38 \mathrm{~Hz}$ (less-more involved hand). 


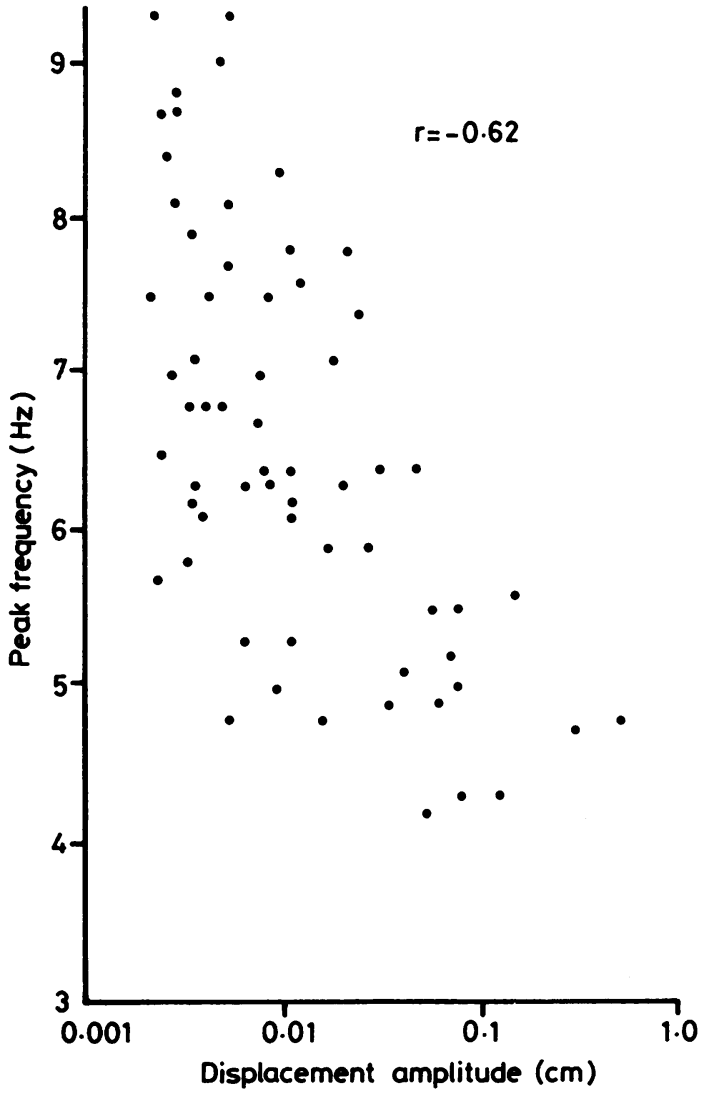

Fig 3 Relationship between dominant peak frequency $(\mathrm{Hz})$ of tremor and log-amplitude displacement (cm) of tremor in the less involved hand in the patients studied $(r=0.62$, $p<0.001)$.

peak frequency $(\Delta f)$ in individual patients $(r=0.33$, $\mathrm{p}<0.005$, fig 4).

Inspection of the plot of $\Delta$ amplitude/ $\Delta$ frequency of tremor in fig 4 indicates that the data points are not distributed normally about the axes. The pattern of distribution at about $0.01 \mathrm{~cm}$ of $\Delta \mathrm{a}$ appears to be different from the pattern below and above this value

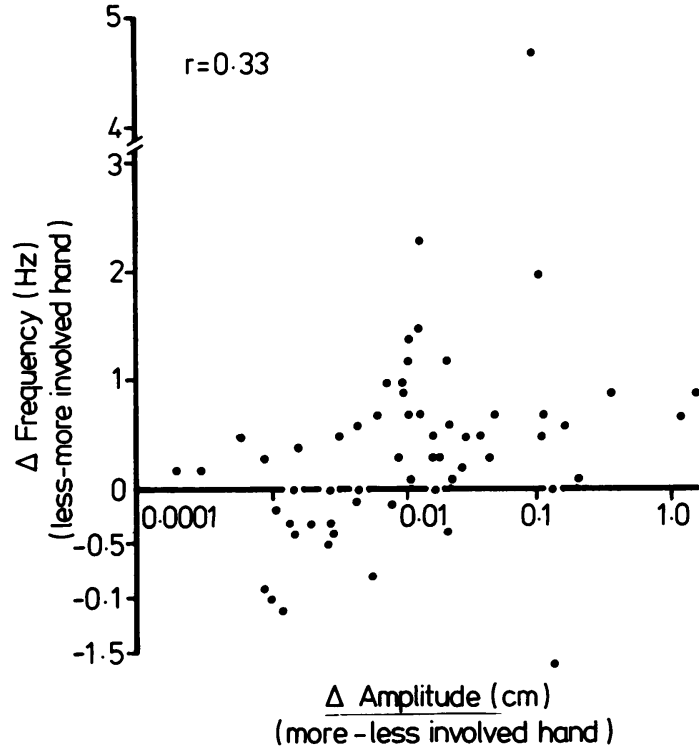

Fig 4 Scatter diagram of difference in frequency between the tremors of the less involved and more involved hand $(\Delta f)$ plotted as a function of the difference in amplitude between the tremors of the less and more involved hand $(\Delta a)$ for each of the patients studied.

which suggests that there are more complex interrelationships between the above variables than simple linear proportionality. Therefore, for further analysis, the population was subdivided into various groups by making experimental cut-offs at tremor amplitudes of $0.005,0.01,0.015,0.02$ and $0.03 \mathrm{~cm}$ hand displacement (see table 3 ). Following this procedure a statistically significant discrimination of three subgroups was shown when cut-off values between 0.01 and $0.015 \mathrm{~cm}$ hand displacement amplitude were chosen $(\mathrm{p}<0.005)$. These subgroups were: group $1(\mathrm{n}=20)$, patients with tremor amplitude in both hands smaller than $0.01-0.015 \mathrm{~cm} ;$ group $2(\mathrm{n}=14)$, patients in whom the tremor in one hand only was less than $0.01-0.015 \mathrm{~cm} ;$ group $3(\mathrm{n}=25)$, patients with tremor in both hands greater than $0.01-0.015 \mathrm{~cm}$.

Table 2 Correlation between patient's characteristics and tremor variables

\begin{tabular}{|c|c|c|c|c|}
\hline & $\begin{array}{l}\text { Tremor amplitude of more } \\
\text { affected hand } \\
\text { (cm displacement) }\end{array}$ & $\begin{array}{l}\text { Tremor amplitude of less } \\
\text { affected hand } \\
\text { (cm displacement) }\end{array}$ & $\begin{array}{l}\text { Peak tremor frequency of } \\
\text { more affected hand }\end{array}$ & $\begin{array}{l}\text { Peak tremor } \\
\text { frequency of less } \\
\text { affected hand }\end{array}$ \\
\hline $\begin{array}{l}\text { Age of patients } \\
\text { Age at onset of tremor } \\
\text { Duration of disorder }\end{array}$ & $\begin{array}{l}0.31 p<0.007 \\
\text { NS } \\
0.35 p<0.001\end{array}$ & $\begin{array}{l}0.34 \mathrm{p}<0.004 \\
\text { NS } \\
0.32 \mathrm{p}<0.001\end{array}$ & $\begin{array}{l}-0.44 p<0.001 \\
N S \\
-0.28 p<0.002\end{array}$ & $\begin{array}{l}-0.42 p<0.001 \\
\text { NS } \\
-0.29 p<0.001\end{array}$ \\
\hline
\end{tabular}

NS indicates no correlation. 
Table 3 Amplitude, frequency, coherence, age and duration of symptoms statistics for the patient subgroups

\begin{tabular}{|c|c|c|c|}
\hline & Group I $(n=20)$ & Group $2(n=14)$ & Group $3(n=25)$ \\
\hline $\begin{array}{l}\text { More involved hand } \\
\text { Tremor amplitude }(\mathrm{cm})(\text { mean } \pm \text { SD) } \\
\text { Tremor peak frequency }(\mathrm{Hz})(\text { mean } \pm \text { SD) } \\
\text { Coherence } R / L \text { hand }(\text { mean } \pm S D)\end{array}$ & $\begin{array}{l}0.0059 \pm 0.002 \\
7 \cdot 21 \pm 1 \cdot 24 \\
0.29 \pm 0.21(n=13)\end{array}$ & $\begin{array}{l}0.048 \pm 0.09 \\
6.15 \pm 0.87 \\
0.42 \pm 0.26(n=9)\end{array}$ & $\begin{array}{l}0.19 \pm 0.3 \\
5 \cdot 51 \pm 1.04 \\
0.36 \pm 0.21(n=24)\end{array}$ \\
\hline $\begin{array}{l}\text { Less involved hand } \\
\text { Tremor amplitude }(\mathrm{cm})(\text { mean } \pm \text { SD) } \\
\text { Tremor peak (mean } \pm \text { SD) } \\
\text { Coherence R/L hand (mean } \pm \text { SD) } \\
\text { Age (years) (mean } \pm \text { SD) } \\
\text { Duration (years) (mean } \pm \text { SD) }\end{array}$ & $\begin{array}{l}0.0038 \pm 0.001 \\
7.12 \pm 1.26 \\
0.23 \pm 0.2(\mathrm{n}=13) \\
45.45 \pm 21.4 \\
7.05 \pm 9.62\end{array}$ & $\begin{array}{l}0.0058 \pm 0.002 \\
7 \cdot 22 \pm 1 \cdot 2 \\
0.21 \pm 0.13(n=9) \\
48 \pm 21.4 \\
11 \cdot 3 \pm 10.44\end{array}$ & $\begin{array}{l}0.042 \pm 0.038 \\
5.87 \pm 1.07 \\
0.26 \pm 0.14(n=24) \\
49.7 \pm 25.7 \\
22.2 \pm 18.72\end{array}$ \\
\hline
\end{tabular}

\section{Amplitude/frequency characteristics}

In group 1 and in group 3, the difference in frequency between the right and the left hands was small. The mean difference in tremor peak frequency (less affected-more affected hand, $m \Delta f$ ) was $-0.09 \mathrm{~Hz}$ in group 1 and $0.35 \mathrm{~Hz}$ in group 3, the latter having a mean difference in tremor amplitude (more affected-less affected hand $\mathrm{m} \Delta \mathrm{a}$ ) about 70 -fold greater than the former $(0.148 \mathrm{~cm}$ in group 3 and 0.002 in group 1). In group 1 amplitude and peak frequency of tremor were not correlated in either hand, whereas a significant negative correlation between the two variables was found in both hands in group $3(\mathrm{r}=-0.47, \mathrm{p}<0.005$ in the more involved and $\mathrm{r}=0.46, \mathrm{p}<0.005$ in the less involved hand). Neither in group 1 nor in group 3 were amplitudes and frequency between the two hands significantly correlated.

In contrast, patients whose tremor amplitude exceeds $0.01-0.015 \mathrm{~cm}$ hand displacement in the more but not in the less affected hand (group $2, n=14$ ) present with features different from the two others. In this group the mean difference in peak frequency of tremor between the two hands was significantly greater than in the others (mean $\mathrm{f} 1 \cdot 1 \mathrm{~Hz}, \mathrm{p}<0.005$ ). This indicates a downward "jump" of the peak frequency of tremor in the more involved hand as compared to the contralateral, despite the mean difference in tremor amplitude between the two hands being of intermediate size, that is, about 3.5 times smaller than in group 3 (mean $\Delta a=0.042$ ). A significant positive relationship between $\Delta \mathrm{a}$ and $\Delta \mathrm{f}(\mathrm{r}=0.7, \mathrm{p}<0.01)$ was found in this group of patients in which the amplitude and peak frequency of tremor were negatively correlated only in the more involved hand $(r=$ $-0.69, \mathrm{p}<0.01)$.

In table 3 the mean dominant peak frequency and amplitude of tremor in the three subgroups of patients, the coherence between the tremors of the two hands, the mean age of the patients and the mean duration of tremor in each group is shown.
Relationship with age and duration of symptoms

In group 1, for either hand, the dominant peak frequency of tremor was negatively correlated with the age of the patients $(r=-0.45, p<0.02$ on the more involved and $r=-0.37, p<0.05$ in the less involved hand). There was no correlation between age of patient and amplitude of tremor in either hand. The duration of the disorder was negatively correlated with peak frequency of tremor only in the less affected hand $(r=-0.38, p<0.05)$ and it was positively correlated with tremor amplitude both in the more and the less involved hand $(r=0.44, p<0.02$ and $r$ $=0.46, \mathrm{p}<0.01$ respectively).

In group 2 there was a negative correlation between the age of the patients and peak frequency of tremor only in the less affected hand $(r=-0.56, p<0.045)$. Patient's age was not significantly correlated with amplitude of tremor in either hand. There was no significant correlation between duration of tremor and frequency or amplitude of tremor for either hand.

In group 3 age of patients and peak frequency of tremor were negatively correlated only in the more affected hand $(r=-0.47, p<0.01)$. Only in the more affected hand was there a positive relationship between age of the patients and amplitude of tremor $(r=0.32, p<0.05)$. Duration of tremor was not correlated with either frequency or amplitude in either hand.

\section{Discussion}

The findings of this study reveal a complex relationship between tremor amplitude, frequency and patient age within individual subjects with ET of differing degrees of severity.

In patients with mild ET (group 1 of our statistical analysis) that is, with tremor amplitude not exceeding $0.01-0.015 \mathrm{~cm}$ hand displacement bilaterally, there was no significant difference in the distribution of frequency of tremors in the right and left hands. The amplitude of tremor was unrelated to frequency, indi- 
cating that they are independent variables. Since in these patients there was a significant negative relationship between age and peak frequency of tremor, it seems that age-related factors affecting the mechanisms of the tremor are the main determinants of the dominant peak frequency of mild ET. A similar negative correlation between age and peak frequency of tremor has been observed for physiological tremor. $^{8}$

In patients belonging to group 2 (tremor amplitude larger than $0.01-0.015 \mathrm{~cm}$ in one hand only), the tremor of the more affected hand is $1 \mathrm{~Hz}$ lower in frequency, on average, than the tremor of the less involved hand. In these patients a significant negative relationship between amplitude and frequency of tremor was found in the more affected hand. This indicates that $0 \cdot 01-0.015 \mathrm{~cm}$ amplitude hand displacement is a critical threshold, above which frequency and amplitude become interdependent in an inverse relationship. This phenomenon has been observed to occur irrespective of the age of the patient or of the peak frequency of tremor in the less involved hand. Therefore, for more severe ET, tremor frequency is more closely related to tremor amplitude than to the patient's age. This finding is in agreement with Elble $^{5}$ who reported that in advanced ET patients " $\log$ amplitude alone accounted for $74 \%$ of total frequency variance, whereas age accounted for an additional $6 \% "$.

In group 3 (tremor amplitude in both hands exceeding $0.01-0.05 \mathrm{~cm}$ ) a trend is shown for peak frequency of tremor to become bilaterally similar although a wide variety of amplitudes of tremor may be found at the same or similar frequencies; thus low frequencies of tremor are compatible with a wide range of tremor amplitudes. This is indirectly supported by the finding that tremorolytic agents (propranolol and primidone), markedly decrease tremor amplitude without affecting peak frequency. In this group, amplitude and peak frequency of tremor are still closely correlated variables, but, in the most severe tremor, patient's age is reestablished as a determining factor of tremor frequency.

The findings in our patients, who represent different stages of ET from mild to severe, relate to current concepts of classification and mechanism in the following way.

Firstly there is "mild" tremor, which in our patients, had an amplitude up to $0.01-0.015 \mathrm{~cm}$ hand displacement. The frequency of this tremor is related to age, as with physiological tremor, and in patients with unilateral symptomatic "mild" tremor has a similar frequency to the physiological tremor in the unaffected hand (data not included in the study). For these reasons the tremor would seem to be derived from mechanisms of normal physiological tremor.
The mechanisms contributing in various proportions to physiological tremor include underdamped limb mechanics and the stretch reflexes, and a tendency for motor unit firing to become synchronised at a discharge rate of about 8 to $12 \mathrm{~Hz}^{5}$ Tremor due to enhanced gain in the peripheral stretch reflex corresponds to type I ET of Marsden et al "benign exaggerated physiological tremor" and is responsive to beta-adrenoreceptor blockade. However, the small amplitude ET in our patients was poorly responsive to acute and sustained administration of propranolol ${ }^{4} 9$ and has been termed "primary", spontaneously occurring, exaggerated physiological tremor" in order to distinguish it from the "secondary" enhanced physiological tremors arising from anxiety stress and beta-adrenergic stimulation. Thus, to retain the classification of Marsden and colleagues, type I ET must be subdivided according to whichever enhanced physiological mechanism is predominantly responsible for the tremor.

If tremor amplitude in the more involved hand is greater than $0.01-0.015 \mathrm{~cm}$ hand displacement then it is $1.1 \mathrm{~Hz}$, on average, lower in frequency than the small amplitude tremor of the less involved hand. The larger amplitude tremor is probably generated by a separate central mechanism. This threshold of tremor amplitude could be assumed as the "break-point" between the type I and type II ET. With further bilateral increase in tremor amplitude the frequencies of tremor on both sides are similar and are down to thes lowest values of frequency encountered clinically.

If familial and sporadic ET are expressions of the same disorder then the results of this study are in agreement with the views of Marsden et $a l^{6}$ and contrast with those of Elble ${ }^{5}$ who claims a continuity between physiological and essential tremor, a component of the former being a forme frustre of the latter. According to Elble, "familial" ET commences with a high frequency tremor $(8-12 \mathrm{~Hz}$ physiological tremor) which gradually decreases in frequency (down to 5-7 Hz), increasing with amplitude, with the progression of the disease. In this scheme there is apparently no obvious "break" in frequency/amplitude characteristic which would suggest the involvement of different types of tremor mechanisms.

The lack of significant degree of coherence between the tremors of the two hands in the patients with ET confirms a previous report. ${ }^{10}$ In most patients there was no significant coherence at the peak frequency of the tremor of either the more or the less involved hand and, in this respect, there were no significant differences between the three subgroups. However, in seven patients there was a significant coherence $(>0.6$ on 150 averages) that was due to passive transmission of tremor from side to side. Passive transmission was 
closely related to tremor amplitude and occurred more frequently with larger tremors $(1 / 13$, group 1 ; $3 / 9$, group $2 ; 3 / 24$, group 3 ). In four patients, showing similar peak frequency and amplitude of tremor in both hands separately, the coherence at the mutual tremor frequency for both hands raised, ranged between 0.4 and 0.8 , suggesting that in some patients the tremors of the right and left hands may be very closely related, possibly sharing a common tremorogenic mechanism or, alternatively that the mechanism of tremor in one hand is somehow driven by the mechanism of tremor in the other hand.

\section{Conclusions}

The results of this study show that age-related factors represent the main determinants of the dominant peak frequency of ET in patients with mild degree of severity of the disorder, and at this stage it may be regarded as a "spontaneously" or "primary" exaggerated form of physiological tremor which arises from a variety of intrinsic central nervous and peripheral neuromuscular factors. Above the limit of tremor amplitude of $0.01-0.015 \mathrm{~cm}$ hand displacement the two main tremor variables, that is, amplitude and dominant peak frequency, become mutually interdependent and the peak frequency of the tremor becomes only secondarily dependent on age related factors. The higher amplitude tremor is probably largely determined by central nervous mechanisms. These two forms of tremor, respectively, mild and more severe, may represent stages of the progression of the disease but there is, as yet, no evidence that the tremor in patients changes from one type to the other.

The authors thank Dr A Mutti of the Istituto di
Clinica Medica e Nefrologia, Cattedra di Medicina del Lavoro, Universita di Parma, for his help in statistical analysis.

\section{References}

1 Larsen TA, Calne DB. Essential tremor. Clin Neuropharmacol 1983;6(3):185-205.

2 Findley LJ, Gresty MA. Tremor. Br J Hosp Med 1981;26:16-32, 181.

3 Shahani BT, Young RR. Action tremors: a clinical neurophysiological review. In: Desmedt JE, ed. Physiological Tremor, Pathological Tremors and Clonus. Basel: Karger, 1978:129-37.

4 Calzetti S, Findley LJ, Perucca E, Richens A. The response of essential tremor to propranolol: evaluation of clinical variables governing its efficacy on prolonged administration. J Neurol Neurosurg Psychiatry 1983;46:393-98.

5 Elble RJ. Physiologic and essential tremor. Neurology 1986;36:225-31.

6 Marsden CD, Obeso A, Rotherwell JC. Benign essential tremor is not a single entity. In: Yahr MD, ed. Current Concepts of Parkinson's Disease and Related Disorders. Amsterdam: Excerpta Medica, 1983:31-46.

7 Gresty MA, Findley LJ. Definition, analysis and genesis of tremor. In: Findley LJ, Capildeo R, eds. Movement Disorders: Tremor. London: The Macmillan Press, 1985:15-26.

8 Marshall J. The effect of ageing upon physiological tremor. J Neurol Neurosurg Psychiatry 1961;24: 145-176.

9 Calzetti S, Findley LJ, Gresty MA, Perucca E, Richens A. Effect of a single oral dose of propranolol in essential tremor: a double-blind controlled study. $\mathrm{Ann} \mathrm{Neu-}$ rol 1983;13:165-71.

10 Barlow JS, Schwab RS. Mutual limb stabilization in relation to tremor characteristics in parkinsonian and essential or heredofamilial tremor. Neurology 1971;21:78-90. 